\title{
Placenta of discordant twins: lack of change in histochemically detectable enzyme activities
}

\author{
S Matsubara, H Minakami and I Sato \\ Department of Obstetrics and Gynecology, Jichi Medical School, Kawachi-gun, Tochigi, Japan
}

\begin{abstract}
We localised three important enzymes histochemically in placental trophoblasts from women who gave birth to dichorionic discordant twins, in which the co-twin was affected by foetal growth restriction (FGR). The enzymes studied were adenosine diphosphate-degrading enzyme (ADPdegrading enzyme, plasma membrane enzyme), cytochrome c oxidase (mitochondrial enzyme), and glucose-6-phosphatase (endoplasmic reticular enzyme). We compared these enzyme activities and their distribution patterns among placentas of the smaller (FGR) co-twin, larger co-twin, preeclamptic singleton with FGR, and normal singletons with birth weight of appropriate for their gestational ages. In FGR co-twin placentas, the intensity and localisation pattern of these three enzymes did not differ from those seen in the larger co-twin and normal singleton placentas. Decreased ADP-degrading activity and cytochrome c oxidase negative mitochondria, which were characteristic features of pre-eclamptic trophoblasts, were not observed in FGR co-twin placentas. These observations indicated that, in the FGR co-twin, enzyme-histochemically detectable trophoblastic cell dysfunction may be absent, or if present, less prominent, compared with preeclamptic FGR. We previously reported that placental trophoblasts from singleton idiopathic FGR also showed no reduction in these enzyme activities. In mechanism and pathophysiology, FGR in dichorionic discordant twins may be quite different from pre-eclamptic FGR, but somewhat resembles idiopathic FGR, though all three disorders lead to placental insufficiency, resulting in limited foetal growth. Twin Research (2000) 3, 123-128.
\end{abstract}

Keywords: placenta, discordancy, enzyme-histochemistry, ADP, cytochrome c oxidase

\section{Introduction}

Weight discordancy in twins with growth restriction in one foetus, is a major cause of perinatal morbidity and mortality in not only monochorionic but also in dichorionic twins. ${ }^{1-3}$ Erkkola et $\mathrm{al}^{2}$ observed that perinatal mortality of discordant twin pairs, defined as a $25 \%$ difference in birth weight, was approximately 2.5 times higher than that of non-discordant ones. Whilst in monochorionic twins the discordancy is mostly attributed to placental vascular communications, ${ }^{1,3}$ the aetiology of discordant dichorionic twins has not been determined. Some factors that cause foetal growth restriction (FGR) in a singleton may also cause a single foetus growth restriction in twins, thus resulting in dichorionic discordant twins. Foetal malformation, chromosomal anomalies, intrauterine infection, and cord anomaly are examples of these factors. ${ }^{4,5}$ In addition to these, one type of FGR exists which is due to a

Correspondence: Shigeki Matsubara, MD, Department of Obstetrics and Gynecology, Jichi Medical School, Minamikawachimachi 3311-1, Kawachi-gun, Tochigi, 329-0498, Japan. Tel: $\quad+81285587376$; Fax: $\quad+81285448505$; E-mail: matsushi @ichi.ac.jp

Received 18 January 2000; revised 24 March 2000; accepted 12 April 2000 common disorder - FGR as a result of preeclampsia.

The detailed mechanism by which pre-eclampsia leads to FGR remains unknown. Recently, however, we have found that activities and subcellular distribution patterns of some important placental enzymes, such as ADP-degrading enzyme (a plasma membrane enzyme ${ }^{6}$ and cytochrome c oxidase (CCO, a mitochondrial enzyme), ${ }^{7}$ changed in placental trophoblasts in patients with severe preeclampsia complicated by FGR. In pre-eclampsia, dysfunction of the placental trophoblasts may be present at each cell level, which may lead to placental insufficiency, finally resulting in FGR. ${ }^{6,7}$ If cytochemically detectable trophoblast dysfunction al so exists in smaller co-twin placenta, similar to the pre-eclamptic placenta, the same mechanism may be functioning in the pathogenesis and pathophysiology in discordant twin FGR and pre-eclampsia. If not, a different mechanism might be present. Does trophoblast cell dysfunction also exist in FGR placenta of dichorionic discordant twins, similar to preeclamptic placenta? In another recent report, we demonstrated the presence of glucose-6-phosphatase (G6Pase, a glucose generating enzyme) in human placental trophoblasts. ${ }^{8}$ Since glucose is the major fuel for foetal growth, reduction or change in G6Pase activity may be expected to occur in FGR co-twin 
placenta. Does placental G6Pase activity change in this circumstance? The present study was the first effort to answer these questions, using ultrastructural enzyme histochemistry.

\section{Materials and methods}

Placentas from 22 pregnant Japanese women were studied. Subjects consisted of the following three groups of women who delivered:

(1) dichorionic discordant twins, defined as a $25 \%$ difference in birth weight, without clinical signs of pre-eclampsia (33-37 weeks' gestation) $(\mathrm{n}=6)$;

(2) singleton FGR babies, having signs of severe pre-eclampsia (30-38 weeks' gestation) $(n=6)$; and

(3) singleton infants appropriate for their gestational ages (AGA) without evidence of preeclampsia (29-38 weeks' gestation) ( $n=10$, normal control).

In discordant twins, all smaller co-twins were light for their gestational ages (FGR; under 1.5 standard deviation (-1.5SD)), defined according to growth standard of the Japanese twin population. ${ }^{9}$ Chorionicity was determined by postpartum histological examination. Among six twin pairs, five pairs were of opposite gender and the remaining one of like-gender. The mother of this like-gender twin received artificial ovulation induction by hMG, followed by gamete intrafallopian transfer (GIFT). Therefore, though we did not perform genotype analysis of the placentas or the infants, the majority in group (1) were considered to be dizygotic twins. In singleton pre-eclamptic pregnancy, FGR was defined as a birth weight under 1.5SD in the Japanese population. ${ }^{10}$ Severe pre-eclampsia was defined according to the criteria of the Japanese Society of Obstetrics and Gynecology as having a maternal blood pressure $\geq 160 / 110 \mathrm{mmHg}$ on at least two separate occasions, or a urinary protein level $\geq 200 \mathrm{mg} / \mathrm{dl}$. All 22 women delivered infants by Caesarean section due to discordant twins (group 1), foetal distress (group 2) and previous Caesarean section or breech presentation with uncontrollable uterine contractions (group 3). None of the infants had any signs of chromosomal anomalies, malformations or congenital infections. Macroscopic observation and routine histological examinations did not reveal the presence of placental or cord abnormalities. There was no significant differences in gestational weeks among the three groups. After obtaining informed consent from all women, several pieces of macroscopically normal placental tissue were taken from the maternal surface within $1 \mathrm{~min}$ of placental delivery. In twins, specimens were taken both from the placenta of the smaller (FGR) infant and that of the larger one. Procedures employed for enzyme histochemistry have been described in detail in our previous papers. ${ }^{6-8}$ In brief, tissues were fixed in $0.5-2.0 \%$ glutaral dehyde solutions in a cacodylate buffer $(\mathrm{pH} 7.4)$ for $30 \mathrm{~min}$ at $4^{\circ} \mathrm{C}$. For the cytochemical detection of the enzyme activities, placental sections of $40 \mu \mathrm{m}$ thickness were incubated at $37^{\circ} \mathrm{C}$ in the following reaction media: lead nitrate medium $^{11-13}$ for ADP-degrading enzyme, diaminobenzidine medium ${ }^{14}$ for CCO, and cerium medium ${ }^{15}$ for G6Pase. Sections then were post-fixed in 1.0\% buffered osmium tetroxide, dehydrated and embedded in epoxy resin. Ultra thin sections were prepared and observed under a transmission electron microscope.

\section{Results}

Electron-dense precipitates indicating ADP-degrading enzyme activity were visible on the apical plasma membranes of the syncytiotrophoblasts (Figure 1a-d). In placentas from pre-eclampsia, the precipitates decreased markedly (Figure 1c), which was in accordance with our previous data. ${ }^{6}$ The precipitates in FGR co-twin placenta were as dense as those in the larger co-twin and normal AGA placenta (Figure 1a,b,d).

CCO staining was localized with mitochondria in trophoblasts (Figure 2a-d, Figure $\left.2 a^{\prime}-d^{\prime}\right)$. In preeclamptic placenta, some mitochondria exhibited positive CCO staining and others did not (Figure2c, $\left.c^{\prime}\right)$, which agreed well with our previous observations. ${ }^{7}$ In the FGR co-twin, all mitochondria were stained for $\mathrm{CCO}$, and no CCO-negative mitochondria were observed (Figure2a, a'). The larger co-twin (Figure 2b, b') and normal singleton AGA placenta (Figure2d, d') did not have CCO-negative mitochondria either.

Precipitates indicative of G6Pase were demonstrated in syncytial endoplasmic reticiulum, as was previously described by us (Figure $3 a-d) .{ }^{8}$ The intensities and distribution were the same among the four specimens (Figure 3a-d). There were no significant differences in staining intensity or staining pattern within the placentas from the same group, nor significant variations in findings among specimens taken from the same placenta. Thus the observations 

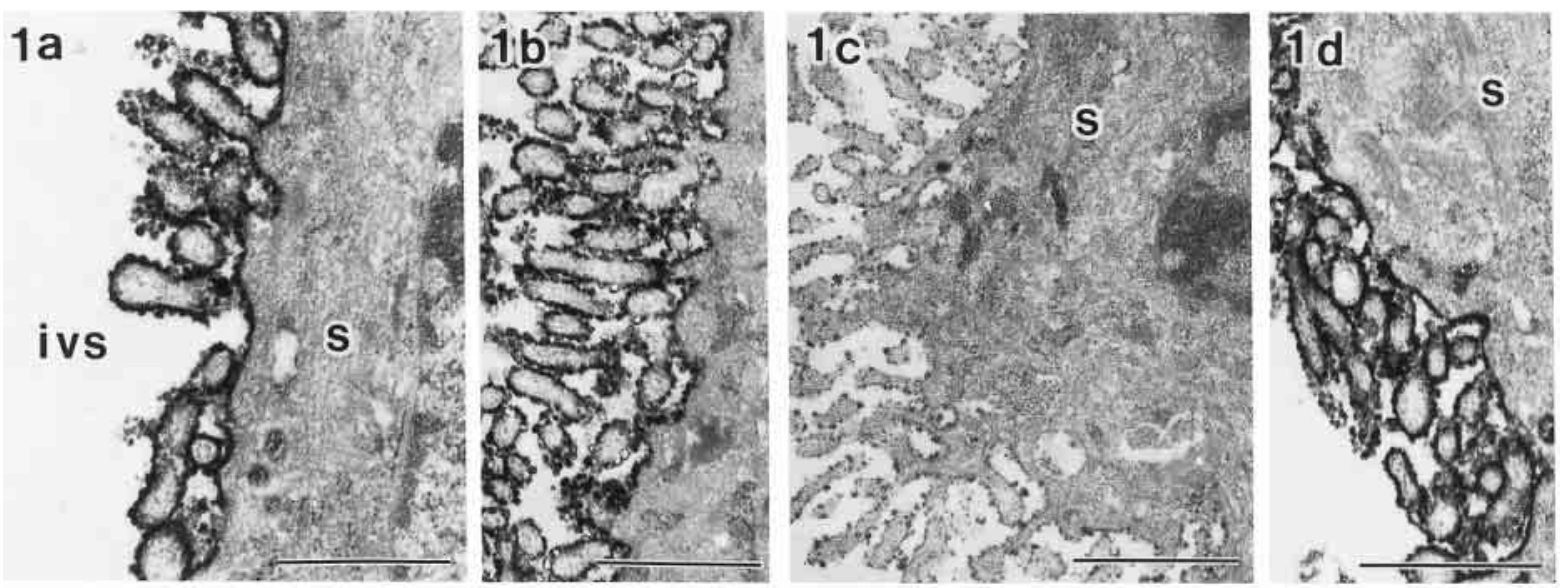

Figure1 ADP-degrading enzyme activities in human placental trophoblasts indicated by electron-dense precipitates visible in the apical plasma membranes. In pre-eclamptic trophoblasts (c), precipitates were significantly weaker than those in FGR co-twin (a), Iarger co-twin (b), and normal AGA singleton placenta (d). Trophoblasts from FGR co-twin (a) exhibited the same enzyme intensity as normal AGA (d). Bar: $1 \mu \mathrm{m}$; ivs: intervillous space; s: syncytiotrophoblast
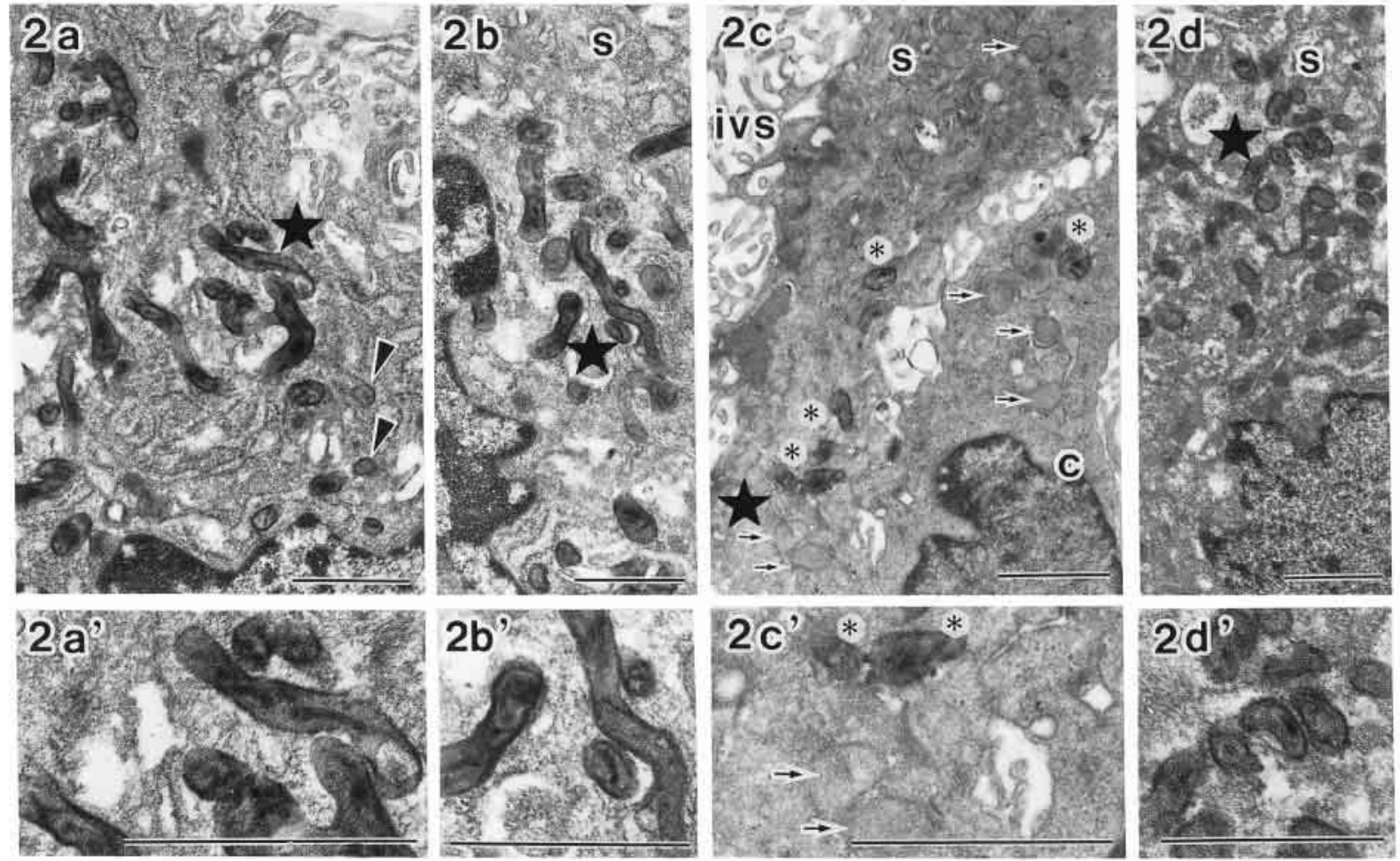

Figure 2 Cytochrome c oxidase (CCO) activity. * areas in a, b, c and d are reproduced at higher magnification in a', b', c' and d'. In FGR co-twin placental trophoblasts (a, $\left.a^{\prime}\right)$, although some mitochondria exhibited weaker staining (arrowheads), all mitochondria stained positive for CCO, similar to those of larger co-twin (b, b') and normal AGA (d, d'). In pre-eclampsia (c, c'), CCO-positive $\left(^{* *}\right)$ and negative (arrows) mitochondria co-existed in syncytiotrophoblast. Pre-eclamptic cytotrophoblast also had both CCO-positive $\left({ }^{*}\right)$ and negative (arrows) mitochondria (c). Bar: $1 \mu \mathrm{m}$; ivs: intervillous space; s: syncytiotrophoblast; c: cytotrophoblast 


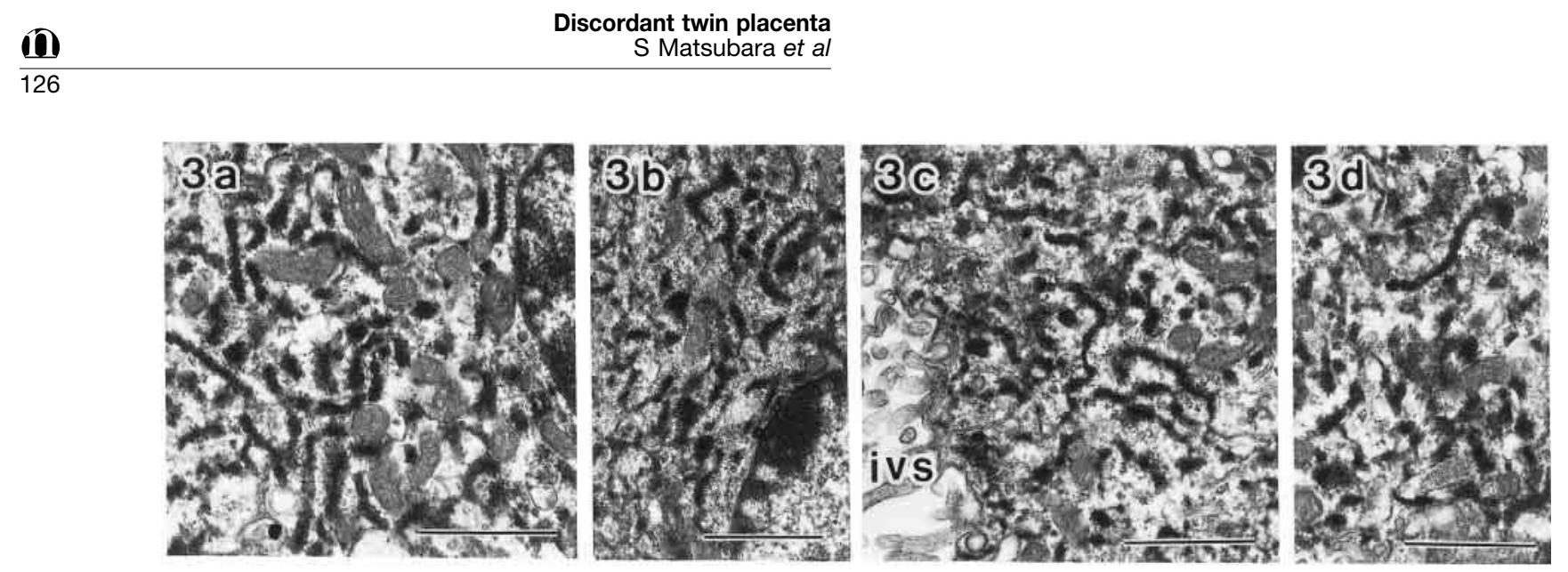

Figure3 Glucose-6-phosphatase (G6Pase) activity. Precipitates were visible in the endoplasmic reticula (a-d). The intensity and distribution were identical among the four specimens. Bar: $1 \mu \mathrm{m}$; ivs: intervillous space

made on these sections were believed to be representative findings of various placental specimens.

\section{Discussion}

FGR in dichorionic twins is quite risky. Recent advances in infertility treatment have increased the occurrence of dichorionic twin gestation, thus the frequency of dichorionic twin FGR has also increased. The perinatal death rate of twins with discordancy was reported to be 2.5 times higher than those without. ${ }^{2}$ A cohort study we performed on the outcome of dichorionic twins indicated a much higher risk. ${ }^{3}$ The risk of adverse outcome was 5.8 times higher in dichorionic pairs with weight discordancy $(\geq 25 \%)$, compared with that in those without. ${ }^{3}$ All the affected foetuses/neonates were the smaller co-twins. These indicated that the FGR co-twin is at high risk, but data highlighting the pathogenesis and pathophysiology are relatively a few. In particular, research into the placental morphological aspect has been lacking. The aetiology of FGR in dichorionic discordant twins remains elusive. Differences in genetic potential, foetal gender, congenital anomalies and anatomical abnormalities of the cord such as velamentous cord insertion have been considered contributing factors in disparate foetal growth between dichorionic twins, ${ }^{1,5,16}$ all of which also affect foetal growth in singleton pregnancy.

One common cause of FGR in singl eton pregnancy is placental insufficiency due to pre-eclampsia. The detailed mechanism of how pre-eclampsia induces FGR remains obscure, but recently we made an important observation concerning this. In placental trophoblasts from pre-eclampsia with FGR, there was a reduction in ADP-degrading enzyme ${ }^{6}$ and CCO activity, ${ }^{7}$ which was also confirmed in the present study. ADP-degrading enzyme, present in apical syncytial membranes facing the intervillous maternal blood, ${ }^{12,13}$ hydrolyses ADP (a potent platelet aggregator) to AMP (an anti-platelet aggregator), protecting the maternal blood against aggregation, thus maintains the microcirculation of the intervillous space. ${ }^{12,13} \mathrm{CCO}$ is a mitochondrial enzyme, which supplies energy within cells. Reduction in these two enzyme activities may impair the placental microcirculation and energy supply within trophoblasts, which may lead to placental insufficiency, and finally to FGR. ${ }^{6,7}$ Thus, in placentas from patients with pre-eclamptic FGR, cytochemically detectable trophoblastic dysfunction does exist at each trophoblast cell level, which may be a causal factor of placental insufficiency and FGR. ${ }^{6,7}$ In the present study, however, enzyme activities and distribution of these two enzymes did not differ between smaller co-twin, larger co-twin, and normal singleton AGA placentas. Reduction in ADP-degrading enzyme activity and occurrence of CCO-negative mitochondria, which were characteristic of preeclamptic trophoblasts, ${ }^{6,7}$ did not occur in FGR co-twin placentas. A different mechanism may be functioning at each trophoblast cell level between placentas of pre-eclampsia and discordant twins, although both placentas accompany limited foetal growth.

Another common cause of FGR is singleton idiopathic FGR. Idiopathic FGR is defined as limited foetal growth without apparent causes, such as preeclampsia, uterine anomaly, foetal malformation etc. FGR co-twin placenta resembles idiopathic FGR placenta, rather than pre-eclamptic placenta, according to the two following morphological observations. First, placentas of smaller co-twin in dichorionic discordant twins exhibited the same macro or microscopic lesions as those observed in singleton idiopathic FGR placentas. ${ }^{16}$ Lesions observed were infarction, abruptio, decidual vasculopathy, villitis, 
intraplacental thrombi, and villous fibrosis. ${ }^{16} \mathrm{Sec}-$ ond, placentas of FGR co-twin and idiopathic FGR exhibited the same subcellular pattern as far as cytochemically detectable enzymes were concerned. ${ }^{17}$ Our recent data ${ }^{17}$ indicated that idiopathic FGR placenta did not exhibit either reduction in ADP-degrading enzymes or appearance of CCOnegative mitochondria (characteristics of preeclamptic placenta), which findings are in complete agreement with those in FGR co-twin placentas observed in the present study. Concerning the morphological features of pre-eclamptic and idiopathic FGR placentas, some previous observations are worth reviewing. Villous structure and intervillous oxygen content are quite different in these two diseases. ${ }^{18-20}$ In pre-eclampsia, there was hypoxia in the intervillous space, leading to syncytial necrosis and cytotrophoblast proliferation, whilst in idiopathic FGR, there was intervillous hyperoxia, leading to reduction in the number of cytotrophoblasts. ${ }^{18-20}$ Although we did not analyse the number of cytotrophoblast nor intervillous oxygen concentration in the present study, there is a high expectation that hyperoxia in the intervillous space will occur in FGR co-twin placentas as well, considering the striking morphological and enzymehistochemical similarities between FGR co-twin and idiopathic FGR placentas. Whilst hypoxia in preeclampsia leads to enzyme dysfunction in syncytium, hyperoxia in the FGR co-twin intervillous space may maintain these placental enzyme activities. Thus, FGR co-twin placentas may not exhibit the reduction in enzyme activities. We do not have direct evidence, so this remains hypothetical but reasonable.

What, then, induces FGR in dichorionic twins? In idiopathic FGR placenta, marked reduction in total trophoblastic surface area, ${ }^{21}$ and structural abnormalities of placental foetal vessels (long, poorly branched foetal capillaries; thus not well functioning vessels) ${ }^{19}$ were reported. These morphological abnormalities are greatly expected to be present in FGR co-twin placentas, considering the structural similarities of these two disorders. Further, as mentioned above, FGR co-twin and idiopathic FGR placentas shared the same macro/microscopic lesions, and both lacked enzyme reduction at each cell level. Thus, we conclude that in FGR co-twin placentas, dysfunction at each trophoblast cell level does not exist, unlike pre-eclamptic placenta. However, this placenta, similar to that in idiopathic FGR, cannot function well as a whole, which may lead to placental insufficiency and finally to FGR. From the morphological perspective, in FGR co-twin placenta, difference from pre-eclampsia, but resemblance to idiopathic FGR, was strongly suggested. Taking all these observations from our research and that of other researchers into account, it was suggested that pathogenesis and pathophysiology of the FGR co-twin may be the same as, or at least resemble, those of idiopathic FGR, but quite different from those of pre-eclampsia.

Recently, we demonstrated that one important placental enzyme, ie G6Pase, existed in human placental trophoblasts, ${ }^{8}$ the presence of which has long been controversial. This enzyme hydrolyzes glucose-6-phosphate to glucose, and thus may play a role in delivering glucose to the foetus. ${ }^{8}$ G6Pase may contribute to foetal growth. In FGR co-twin, foetal hypoglycaemia was reported to be present, ${ }^{22}$ thus change or reduction in G6Pase enzyme activity may be expected to occur. However, there was no reduction in cytochemically detectable G6Pase activity in FGR co-twin placentas. Pre-eclamptic trophoblasts al so exhibited the same distribution pattern for this enzyme. Placental glucose production by G6Pase may remain intact in these FGR placentas.

The detailed mechanism of limited foetal growth in pre-eclampsia and idiopathic FGR is yet unknown. Thus, even though in the present study we characterised cytochemically detectable enzyme activities in FGR co-twin placentas, and thus shed light on the enzyme-histochemical similarities or differences among placentas with these three disorders, the question remains to be solved how FGR occurs in dichorionic discordant twins. Further study on a large population, using methods other than enzyme histochemistry (biochemistry, molecular biology, and other techniques) would be necessary to elucidate this important issue.

\section{References}

1 Cunningham FC, MacDonald PC, Gant NF, Leveno KJ, Gilstrap LC, Hankins GDV, Clark SL. Multifetal pregnancy. In: Cunningham FG, MacDonald PC, Gant NF, Leveno KJ, Gilstrap LC, Hankins GDV, Clark SL (eds). Williams Obstetrics, 20th edn. Appleton \& Lange: Connecticut, 1997, pp 861-894.

2 Erkkola R, Ala-Mello S, Piiroinen O, Kero P, Sillanpaa M. Growth discordancy in twin pregnancy: a risk factor not detected by measurements of biparietal diameter. Obstet Gynecol 1985; 66: 203-206.

3 Minakami H, Honma Y, Matsubara S, Uchida A, Shiraishi H, Sato I. Effects of placental chorionicity on outcome in twin pregnancy, a cohort study. J Reprod Med 1999; 44: 595-600.

4 Robinson JS, Owens J. The placenta and intrauterine fetal growth retardation. In: Redman CWG, Sargent IL, Starkey PM (eds). The Human Placenta. Blackwell: Oxford, 1993, pp 558-578.

5 Benirschke K, Kaufmann P. Anatomy and pathology of the umbilical cord and major fetal vessels. In: Benirschke K, Kaufmann P (eds). Pathology of the Human Placenta. Springer-Verlag: New York 1995, pp 319-377.

6 Matsubara S, Sato I, Saito T. Ultrastructural Iocalization and cytochemical characteristics of human placental ADP- degrading activity in normal and preeclamptic pregnancy. Troph Res 1997; 9: 121-129. 
7 Matsubara S, Minakami H, Sato I, Saito T. Decrease in cytochrome c oxidase activity detected cytochemically in the placental trophoblasts of patients with pre-eclampsia. Placenta 1997; 18: 255-259.

8 Matsubara S, Takizawa T, Sato I. Glucose-6-phosphatase is present in normal and pre-eclamptic placental trophoblasts: ultrastructural enzyme-histochemical evidence. Placenta 1999; 20: 81-85.

9 Fukuda M. Clinical study of twins, intrauterine growth curves in twins. Acta Neonat Jpn 1990; 26: 366-371.

10 Nishida H, Sakanoue M, Kurachi K, Asada A, Kudo S, Funakawa $\mathrm{H}$. Fetal growth curve of Japanese. Acta Neonat Jpn 1984; 20: 90-97.

11 Novikoff AB, Goldfischer S. Nucl eotide diphosphatase activity in the Golgi apparatus and its usefulness for cytochemical study. Proc Natl Acad Sci USA 1961; 47: 802-810.

12 Matsubara S, Tamada T, Kurahashi K, Saito T. Ultracytochemical localization of adenosine nucleotidase activities in the human term placenta, with special reference to 5'-nucleotidase activity. Acta Histochem Cytochem 1987; 20: 409-419.

13 Matsubara S, Tamada T, Saito T. Ultracytochemical Iocalization of ADP-degrading enzyme activity in the human term placenta - direct cytochemical evidence. Acta Obst Gynaec Jpn 1987; 39: 2195-2196.

14 Seligman AM, Karnovsky MJ, Wasserkrug HL, Hanker JS. Nondroplet ultrastructural demonstration of cytochrome oxidase activity with a polymerizing osmiophilic reagent, diaminobenzidine (DAB). J Cell Biol 1968; 38: 1-14.
15 Robinson $\mathrm{M}$, Karnovsky MJ. Ultrastructural localization of some phosphatases with cerium. JHistochem Cytochem 1983; 31: 1197-1208.

16 Eberle AM, Levesque D, Vintzileous AM, Egan JFX, Tsapanos V, Sal afia CM. Placental pathology in discordant twins. Am J Obstet Gynecol 1993; 169: 931-935.

17 Matsubara S, Sato I. Placenta of idiopathic fetal growth restriction: cytochemical ly detectable enzyme activities do not change at a subcellular level. Placenta 2000; 21: 257-262.

18 Benirschke K, Kaufmann P. Maternal diseases complicating pregnancy: diabetes, tumors, pre-eclampsia, Iupus anticoagulant. In: Benirschke K, Kaufmann P (eds). Pathology of the Human Placenta. Springer-Verlag: New York, 1995, pp 476-536.

19 Benirschke K, Kaufmann P. Three-dimensional aspects of villous maldevel opment. In: Benirschke K, Kaufmann P (eds). Pathology of the Human Placenta. Springer-Verlag: New York, 1995, pp 167-181.

20 Macara L, Kingdom JCP, Kaufmann P, Kohnen G, Hair J, More IAR, LyalI F, Greer IA. Structural analysis of placental terminal villi from growth-restricted pregnancies with abnormal umbilical artery doppler waveforms. Placenta 1996; 17: 37-48.

21 Teasdale F, Jean-Jacques G. Intrauterine growth retardation: Morphometry of the microvillous membrane of the human placenta. Placenta 1988; 9: 47-55.

22 Reisner SH, Forbes AE, Cornblath $\mathrm{M}$. The smaller twins and hypoglycemia. Lancet (i) 1965; 542. 